\title{
A Novel Cooperative Spectrum Sharing Algorithm Based on Optimal Cognitive Radio User Selection
}

\author{
Mohammad Bigdeli, Bahman Abolhassani \\ School of Electrical Engineering, Iran University of Science and Technology (IUST), Tehran, Iran \\ Email:1lVllohammad@gmail.com, abolhassani@iust.ac.ir
}

Received October 11, 2011; revised December 7, 2011; accepted December 24, 2011

\begin{abstract}
In this paper, we consider the problem of cognitive radio (CR) user selection to maximize overall CR network (CRN) throughput when the available spectrum bandwidth is less than the demand by all CR users. We formulate optimal CR user selection problem. Then, based on approximation of the average received signal to interference plus noise ratio $(\overline{S I N R})$ and adaptive modulation and coding (AMC), we estimate the required bandwidth of CR users with different required quality of services (QoSs). Using the principle of optimality, we propose a novel cooperative spectrum sharing algorithm for a CRN. The proposed algorithm not only achieves exhaustive search performance but also its complexity is in the order of $N \times M$ versus $2^{N}$ for exhaustive search, where $N$ is the number of CR users, and $M$ is the spectrum pool size. Extensive simulation results illustrate that the proposed algorithm significantly outperforms the existing algorithms that ignore optimal CR user selection. Also, these results illustrate a better fairness criterion than those of previous works.
\end{abstract}

Keywords: Cognitive Radio Network (CRN); Spectrum Sharing; Spectrum Pool; Principle of Optimality

\section{Introduction}

In the fixed spectrum allocation policy that is currently used in wireless networks, fixed spectrum segments are allocated to spectrum-licensed primary radio (PR) users for a long time in large regions. In the last three decades, spectrum demands for the wireless applications have been increased so rapidly such that spectrum scarcity has become a bottleneck of this fixed spectrum allocation policy, while, PR users underutilize significant portions of the allocated spectrums, ranging from $15 \%$ to $85 \%$ of times or regions [1]. The underutilization and the limitation of the frequency spectrum necessitate applying a dynamic spectrum allocation policy, which can be implemented using cognitive radio (CR) technology [2]. Based on dynamic spectrum allocation, in a CR network $(\mathrm{CRN})$, unlicensed-spectrum CR users can use parts of spectrum (spectrum holes) that are not being utilized by PR users at particular times or in specific regions. In a $\mathrm{CRN}$, one of the main components is the spectrum sharing algorithm that is allocating new spectrum holes to $\mathrm{CR}$ users.

A spectrum sharing algorithm is designed with a specific objective. This can be achieved by a cooperative or noncooperative algorithm. In the first case, all CR users cooperatively implement an algorithm such that they optimize overall objective of the CRN. In contrast, CR users may have opportunistic and selfish behaviors in noncooperative spectrum sharing algorithms. Thus, the difference of these two types of spectrum sharing algorithms is in overall or individual definition of the objective. The objectives of spectrum sharing algorithms are throughput, power, interference, profit, and etc. [3]. In this paper, we propose a novel optimal cooperative spectrum sharing algorithm with overall CRN throughput as its objective.

The concept of "spectrum pooling" is the idea of merging different spectrum segments from different PR users into a common spectrum pool, where first was proposed in [4] for cooperative spectrum sharing. In [5], authors used this concept to formulate a cooperative spectrum sharing algorithm as a restless bandits model based optimization system, which maximizes the price and spectrum efficiency based utility function objective. In [6], relationship between the optimal capacity of spectrum pool and network efficiency is studied and a quantitative mathematical relation was derived for it. A basic spectrum sharing algorithm for CRN with a single PR user and multiple CR users was introduced in [7], and an analytical model was implemented to derive the statistics of the spectrum sharing algorithm. Also, in [8], a hierarchical spectrum sharing algorithm was proposed for clustering CRN; the methodologies behind its levels are the list-coloring and maximum matching. Such an algo- 
rithm achieves suboptimal performance.

In [9], authors developed a distance-dependent medium access control protocol (DDMAC) to improve the overall CRN throughput using a cooperative spectrum sharing. This algorithm exploits the dependence between the signal attenuation model and the transmission distance. It assigns spectrum holes with lower average signal to interference and noise ratio $(\overline{S I N R})$ to shorter transmission distances to increase the number of simultaneous transmissions, and consequently, to increases the overall CRN throughput. However, when available spectrum is insufficient to serve all CR users, this algorithm has no idea about how to select CR users in order to achieve optimal overall CRN throughput.

In this paper, we propose a novel optimal cooperative spectrum sharing algorithm that has the ability to select the CR users in order to achieve optimal overall CRN throughput. We use principle of optimality [10]. According to the principle of optimality, an optimal solution of a problem has the property that includes optimal solution for all sub problems of a main problem. We used orthogonal frequency-division multiplexing (OFDM) based spectrum pool, which has high flexibility with respect to spectrum holes [5]. In order to avoid corrupting the transmissions of PR users, a power mask is enforced on the transmission of CR users. The log-distance path loss model is used to approximate the transmission path loss, and $\overline{S I N R}$. Then, we apply these and an adaptive modulation and coding (AMC), to estimate minimum required bandwidths of $\mathrm{CR}$ users. We formulated this optimal user selection problem with the objective of maximizing overall CRN throughput and meeting constraint of the given spectrum pool size. Then, using principle of optimality, we investigate a dynamic form of this problem, and propose a novel algorithm for spectrum sharing.

The proposed algorithm has reasonable (polynomial) computational complexity, which makes it useful for practical networks when spectrum holes are available only for idle period of PR users. In other words, the proposed algorithm not only achieves exhaustive search performance, i.e. optimal solution, but also has low computational complexity. Also, in performance analysis of the proposed algorithm, we consider fairness of the proposed algorithm compared with other algorithms.

The rest of this paper is organized as follows. Section 2 presents preliminaries of the proposed algorithm; includes an overview of the OFDM based spectrum pool and system model. The analysis of bandwidth requirement is presented in Section 3. In Section 4, we formulate the optimal CR user selection problem. Section 5 introduces our proposed cooperative spectrum sharing algorithm. Complexity analysis is given in Section 6. We evaluate the proposed algorithm through simulations in Section 7. Conclusions are given in Section 8.

\section{Preliminaries}

\subsection{OFDM Based Spectrum Pool}

Spectrum pooling is the idea of merging different spectrum segments from PR users into a common spectrum pool [4]. When, some of the PR users are idle, there are spectrum holes in their corresponding spectrums at the common spectrum pool. The CR users may use these spectrum holes of the spectrum pool, during idle periods of PR users. The main proposition is that the operations of CR users must be transparent for PR users, and PR systems do not need to be changed. So, the CRN system must have high flexibility to fill the spectrum holes in the common spectrum pool. In other words, the CR user must jumps from a newly occupied part of the spectrum pool to an unoccupied spectrum from already available separated spectrum holes. OFDM is the best choice for implementing this high flexible CRN.

The key advantage of the OFDM is that a set of subcarriers represented by their corresponding IFFT inputs can be fed with zeros, thus sparing certain spectral areas from the emission of power. So, if the CR user only uses the subcarriers lying in idle spectrums of the PR network (i.e., in the spectrum holes of the spectrum pool), spectral coexistence of both PR and CR systems is possible and the CR users have high flexibility respect to the spectrum holes. In fact, the basic idea of the OFDM based spectrum pool is to match the bandwidth of the PR user's band with an integer multiple of the carrier that used in the CR systems.

\subsection{System Model}

In this paper, we consider a CRN with $N$ CR users who use $L$ different services having different quality of services (QoSs). $\alpha^{l}$ denotes percentage of the CR users in the class $l, 1 \leq l \leq L$. Class $l$ of service type is determined by $R^{l}$ and $B E R_{\text {tar }}^{l}$, respectively, which denote minimum data rate and target bit error rate $(B E R)$ to guarantee minimum quality of service of this class.

The available spectrum in the common spectrum pool is divided into $M$ subchannels, each having a fixed bandwidth $\Delta f$. The number of available subchannels varies by changing size of the spectrum pool. In our proposed algorithm, the spectrum is allocated with the unit of one subchannel.

The CR user's arrival and departure processes in the CRN are considered to be Poisson processes [4]. The expected arrival and departure rates of the CR users are represented by $\lambda_{C R U s}$ and $\mu_{C R U s}$, respectively. Similarly, the arrival and departure processes of available spectrum unit in the spectrum pool are also considered to be Poisson processes, with expected arrival and departure rates $\lambda_{\text {spec }}$ and $\mu_{\text {spec }}$, respectively. Table 1 presents system parameters and their definitions. 
Table 1. Summary of system parameters and their notations.

\begin{tabular}{cc}
\hline Notation & Description \\
\hline$\alpha^{l}$ & Ratio of the CR users in the class $l$ \\
$L$ & Number of available service type's classes in CRN \\
$B E R_{\text {tar }}^{l}$ & Minimum BER required for CR users in class $l$ \\
$R^{l}$ & Target data rate required for CR users in class $l$ \\
$M$ & Number of spectrum units in common spectrum pool \\
$\lambda_{\text {CRU }}$ & Expected arrival rate for CR user \\
$\mu_{C R U}$ & Expected departure rate for CR user \\
$\lambda_{\text {spec }}$ & Expected arrival rate for available spectrum unit \\
$\mu_{\text {spec }}$ & Expected departure rate for available spectrum unit \\
$B_{j}$ & Required spectrum units for $j$ th CR user \\
$R_{j}$ & Required data rate for $j$ th CR user \\
$x_{j}$ & Binary variable equal to 1 if $j$ th CR user is selected \\
$N$ & by spectrum sharing algorithm, otherwise equal to 0 \\
$d_{f}$ & Number of CR users \\
$d_{0}$ & Far field distance from CR transmitter antenna \\
\hline$P L(d)$ & Close in distance from CR transmitter antenna \\
$D_{a}$ & Average path loss at transmission distance $d$ \\
$P_{t}$ & Maximum linear dimension of transmitter antenna \\
$k_{j}^{s}$ & CR transmitter antenna power \\
\hline & Spectrum efficiency for $j$ th CR user \\
\hline
\end{tabular}

\section{CR User Bandwidth Requirement Evaluation}

In this section, we explain how to estimate the bandwidth required for the $j$ th $\mathrm{CR}$ user. First, we evaluate average path loss and $\overline{S I N R}$ experienced by the CR user. Then, using AMC, we estimate the bandwidth required for the $\mathrm{CR}$ user. These estimations of required bandwidths will be used by the proposed spectrum sharing algorithm presented in Section 5.

\subsection{Pass Loss Evaluation}

We use the log-distance path loss model to estimate the path loss in the CRN [11]. This model is only valid for distances beyond the far field distance from the $\mathrm{CR}$ transmitter antenna, $d_{f}$, which depends on the maximum linear dimension of transmitter antenna $\left(D_{a}\right)$, and carrier wavelength $(\lambda=c / f$, where $c$ is the speed of light), and can be estimated as [12]

$$
d_{f}=\max \left\{D_{a}, \lambda, \frac{2 D_{a}^{2}}{\lambda}\right\}
$$

$\overline{P L}(d)$ denotes the average large-scale path loss between a transmitter and a receiver separated by distance $d$. By choosing the close-in distance $d_{0}\left(d_{0} \geq d_{f}\right)$ as a reference distance; based on the log-distance path loss model, $\overline{P L}(d)$ for $d \geq d_{0}$ (at far field distance) is expressed as

$$
\overline{P L}(d)=\overline{P L}\left(d_{0}\right)\left(\frac{d}{d_{0}}\right)^{n},
$$

where $n$ is the path loss exponent (typically, $2 \leq n \leq 6$ ) that indicates the rate at which the path loss increases with $d$, also, $\overline{P L}\left(d_{0}\right)$ is the path loss at $d_{0} . \overline{P L}\left(d_{0}\right)$ can be measured or estimated using Friis propagation model [12].

It is notable that in practice $d_{0}$ is in the order of the $\mathrm{CR}$ transmitter antenna dimension. For example, for a cellular phone operating at $900 \mathrm{MHz}$ band with $D_{a}=5 \mathrm{~cm}$, $d_{f}$ is $33 \mathrm{~cm}$. Consequently, the probability that two CR users being separated by a distance lower than $d_{0}$ is very small (i.e. $\operatorname{Pr}\left(d<d_{0}\right) \approx 0$ ).

\subsection{Average $S I N R$}

For transmission between two CR users, which are separated by $\mathrm{d}$, the $\overline{S I N R}$ at the $\mathrm{CR}$ user receiver antenna over a band is determined by transmitter antenna power $\left(P_{t}\right)$, average path loss associated with that transmission that is expressed using Equation (2), average interference in receiver $(\bar{I})$, and thermal noise $\left(\sigma_{n}^{2}\right)$. Formally, $\overline{S I N R}$ is given by

$$
\begin{aligned}
\overline{\operatorname{SINR}}[\mathrm{dB}]= & P_{t}(d)[\mathrm{dBm}]-P L(d)[\mathrm{dB}], \\
& -\bar{I}[\mathrm{dBm}]-\sigma_{n}^{2}[\mathrm{dBm}]
\end{aligned}
$$

where $\bar{I}$ is the inter carrier interference (ICI) of PR users that is evaluated in [13].

\subsection{AMC and CR User Bandwidth Requirment}

Equation (3) is used to estimate $\overline{\text { SINR }}$ (of the CR user transmission) and bandwidth requirement as explained in the following. The CR users employ adaptive modulation and coding to transmit on assigned spectrum holes. For QAM modulation with quadratic constellation, i.e. 4QAM, 16QAM, and etc., BER for a single input single output (SISO) channel with additive white Gaussian noise (AWGN) is expressed as [14]

$$
B E R \approx 0.2 \exp \left(\frac{\overline{S I N R}}{2^{k^{s}}-1}\right),
$$

where $k^{s}[\mathrm{~b} / \mathrm{s} / \mathrm{Hz}]$ is the spectrum efficiency. To guarantee the quality of service for the $j$ th $C R$ user in class $l(1 \leq$ $l \leq L$ ), we set $B E R$ in Equation (4) equal to $B E R_{\text {tar }}^{l}$, and replace $\overline{\operatorname{SINR}}$ in Equation (4) with the R.H.S of Equation (3). Consequently, the required spectrum efficiency $\left(k_{j}^{s}\right)$ for this CR user is expressed as

$$
k_{j}^{s}=\log _{2}\left(1+K^{l} \times \frac{P_{t}(d)}{\overline{P L}(d) \times \bar{I} \times \sigma_{n}^{2}}\right),
$$

where 


$$
K^{l}=\frac{1.5}{\ln \left(0.2 / B E R_{\text {tar }}^{l}\right)}
$$

Thus, the spectrum efficiency, which is required to guarantee minimum quality of service for the $j$ th $\mathrm{CR}$ user (from class $l$ ) is given by Equation (5). As well, having $k_{j}^{s}$ and $R^{l}$, the required bandwidth, $b_{j}$, can be evaluated as

$$
b_{j}=R^{l} / k_{j}^{s}
$$

Thus, the bandwidth requirement of a $\mathrm{CR}$ user is mainly a function of its service type class (i.e. $R^{l}$ and $B E R_{\text {tar }}^{l}$ ), transmission distance, and $\overline{\text { SINR }}$ at its receiver.

As mentioned before, the CRN employs OFDM system, thus, spectrum sharing algorithm had better assign integer multiple of spectrum units to each CR user (if CR user is selected in spectrum sharing algorithm that is explained in Section 5). So, it is appropriate to express $b_{j}$, given by Equation (7), as an integer number of spectrum units. Since, $b_{j}$ expresses the minimum spectrum requirement, the minimum number of required spectrum units is expressed as

$$
B_{j}=\left\lceil\frac{b_{j}}{\Delta f}\right\rceil,
$$

where $\lceil$.$\rceil is the ceil function.$

\section{Optimal CR User Selection Problem}

In many cases, the available spectrum units, $M$ (spectrum pool size), is less than the CR users' bandwidth requirements i.e. $\sum_{i=1}^{N} B_{j} \geq M$. So, the spectrum sharing algorithm must have a strategy in deciding which CR users should use the available spectrum, and which spectrum units should be assigned to which CR users. The decision strategy is designed to achieve a certain objective in the $\mathrm{CRN}$. In this paper, the objective is to achieve the optimal overall CRN throughput. All CR users cooperate to maximizing the overall CRN throughput by selecting a subset of the CR users that have better conditions to use spectrum. The constraint of this optimization problem is the spectrum pool size. Let $R_{j}$ denotes the required data rate for the $j$ th $\mathrm{CR}$ user $(j \in\{1, \cdots, N\})$, that is equal to $R^{l}$ when this user is in class $l$.

The optimization problem of CR user selection with overall CRN throughput objective is: given a set of $N \mathrm{CR}$ users, and a common spectrum pool with $M$ spectrum units, select a subset of the CR users such that

$$
\text { Maximize } \quad \sum_{j=1}^{N} x_{j} R_{j},
$$

subject to

$$
\begin{array}{r}
\sum_{j=1}^{N} x_{j} B_{j} \leq M, \\
x_{j}=0 \text { or } 1, j \in\{1,2, \cdots, N\},
\end{array}
$$

where

$$
x_{j}=\left\{\begin{array}{lr}
1, \text { if } j \text { th } \mathrm{CR} \text { user selected } \\
0, & \text { otherwise }
\end{array}\right.
$$

But, the above integer linear programming (ILP) problem belongs to the class of NP-hard problems. To explain this proposition, we note that to solve this problem a naive approach would be to examine all possible binary vectors $X=\left\{x_{j}, 1 \leq j \leq N\right\}$, selecting the best of those which satisfies the constraints. Unfortunately, the number of such vectors is $2^{N}$, by exhaustive search, even a hypothetical computer, capable of examining one billion vectors per second, would require more than 30 years for a CRN with $60 \mathrm{CR}$ users. However, it is clear that time access to spectrum hole, idle period of PR user, is so lower. On the other hand, the computational complexity of spectrum sharing algorithm should be low because a long computation time may result in the out of date spectrum state information. In Section 5, using principle of optimality, we propose a novel cooperative algorithm that provides an optimal solution that has a reasonable computation time such that in a CRN with $N=100 \mathrm{CR}$ users, optimal solution is obtained in a reasonable time by a regular processor.

\section{Proposed Cooperative Spectrum Sharing Algorithm}

In this section, we propose a novel cooperative spectrum sharing algorithm such that it not only achieves optimal solution but also it has low computational complexity. The decision strategy of this algorithm is a new approach in selecting CR users for maximizing the overall CRN throughput.

In Section 4, we defined the optimization problem, and stated that evaluating optimal solution leads to an exhaustive search with decision tree with $2^{N}$ nodes where it is impractical for spectrum sharing applications from computational complexity view. The principle of optimality where is said to be applied in an optimal solution to an instance of a problem always contains optimal solutions to all sub instances, is used in our proposed algorithm. Based on this principle, we solve our optimization problem for the CRN with small number of $\mathrm{CR}$ users, store these results, and later, whenever we need a result, we look up in previously stored results instead of recomputing it.

We use notation $\psi\left(k_{1}, k_{2}, m\right)$ for the following optimal cooperative spectrum sharing problem: Select vector $X$ such that it maximizes

$$
\sum_{j=k_{1}}^{k_{2}} x_{j} R_{j}
$$

where

$$
x_{j}=0 \text { or } 1 \text {, }
$$




$$
j \in\left\{k_{1}, k_{1}+1, \cdots k_{2}\right\}
$$

subject to

$$
\sum_{j=k_{1}}^{k_{2}} x_{j} B_{j} \leq m
$$

Now, in order to design our cooperative spectrum sharing algorithm we define and prove the following lemma.

Lemma: The cooperative spectrum sharing problem can be expressed in a dynamic form using principle of optimality.

Proof:

We assume $\left\{y_{k_{1}}, y_{k_{1}+1}, \cdots, y_{k_{2}}\right\}$ is an optimal solution $\left(y_{i}=0\right.$ or $\left.1, k_{1} \leq i \leq k_{2}\right)$ for $\left\{x_{k_{1}}, x_{k_{1}+1}, \cdots, x_{k_{2}}\right\}$ i.e. for $\psi\left(k_{1}, k_{2}, m\right)$, where expresses which CR users can use the spectrum. If $y_{k_{1}}=0$, then $\left\{y_{k_{1}+1}, \cdots, y_{k_{2}}\right\}$ must be the optimal solution for $\psi\left(k_{1}+1, k_{2}, m\right)$, otherwise $\left\{y_{k_{1}}, y_{k_{1}+1}, \cdots, y_{k_{2}}\right\}$ is not optimal solution for $\psi\left(k_{1}, k_{2}, m\right)$. On the other hand, if $y_{k_{1}}=1$, then $\left\{y_{k_{1}+1}, \cdots, y_{k_{2}}\right\}$ must be optimal solution for $\psi\left(k_{1}+1, k_{2}, m-B_{k-1}\right)$, otherwise we have another sequence such as $\left\{z_{k_{1}+1}, \cdots, z_{k_{2}}\right\}$ which satisfies

$$
\sum_{j=k_{1}+1}^{k_{2}} z_{j} B_{j} \leq m-B_{k_{1}}
$$

and

$$
\sum_{j=k_{1}+1}^{k_{2}} z_{j} R_{j}>\sum_{j=k_{1}+1}^{k_{2}} y_{j} R_{j}
$$

So, sequence $\left\{y_{k_{1}}, z_{k_{1}+1}, \cdots, z_{k_{2}}\right\}$ is better than optimal solution where it is against the assumption. Consequently, if $V_{k_{1}, k_{2}}^{m}$ denotes an optimal solution value for $\psi\left(k_{1}, k_{2}, m\right)$, i.e.

$$
\begin{gathered}
V_{k_{1}, k_{2}}^{m}=\max \left\{\sum_{j=k_{1}}^{k_{2}} x_{j} R_{j}: \sum_{j=k_{1}}^{k_{2}} x_{j} B_{j} \leq m .\right. \\
\left.x_{j}=0 \text { or } 1, j=k_{1}, \cdots, k_{2}\right\}
\end{gathered},
$$

can be expressed in a dynamic form as follows,

$$
V_{k_{1}, k_{2}}^{m}=\max \left\{V_{k_{1}+1, k_{2}}^{m}, V_{k_{1}+1, k_{2}}^{m-B_{k_{1}}}+R_{k_{1}}\right\} \text {. }
$$

Therefore, the optimal solution for cooperative spectrum sharing problem that is explained in IV is $\psi(1, N, M)$, where we can evaluate its optimal value, $V_{1, N}^{M}$, in a dynamic form using Equation (19). Algorithms 1 and 2 are consecutively are run to obtain the optimal solution for this cooperative spectrum sharing problem. The output of Algorithm 2 is vector $X$ which indicates which CR users are selected for data transmission. Specifically, $j$ th CR user is selected if $x_{j}=1$. We note that the solution obtained from in Algorithm 1 (for the CRN with $N$ CR users and spectrum pool with $M$ spectrum units); it includes the optimal solution for any CRN with $n(n \leq N)$ CR users, and spectrum pool with $m$ spectrum units $(m \leq M)$. This is a property that directly arises from the principle of optimality.

When Algorithm 2 selects the best sub set of CR users that they maximize the overall CRN throughput, spectrum sharing algorithm starts the spectrum allocation step. In this step, we use DDMAC [9], which assigns the spectrum units with high $\overline{S I N R}$ values for near distance transmission and vice versa.

\section{Complexity Analysis}

As mentioned earlier, computational complexity of the cooperative spectrum sharing algorithm must be reasonable such that the CR users have enough time to use spectrum holes. So, it is useful to analyze computational complexity of the proposed algorithm by studying Algorithm 1 and Algorithm 2. In Algorithm 1, computational complexity of initialization steps are in order of $N$ and $M$. Also, computational complexity of two loops in lines two and three of the algorithm is in the order of $N \times M$. So, computational complexity of Algorithm 1 is in the order of $N \times M$. Similarly, complexity of Algorithm 2 is in the order of $N \times M$. Consequently, complexity of the proposed spectrum sharing algorithm is $O(N \times M)$ versus $O\left(2^{N}\right)$ for an exhaustive search of full decision tree. For example, in CRN with $N=40 \mathrm{CR}$ users and spectrum pool with $M=200$; computational complexity is 8000 versus $2^{40}$ respectively for the proposed algorithm and full exhaustive search.

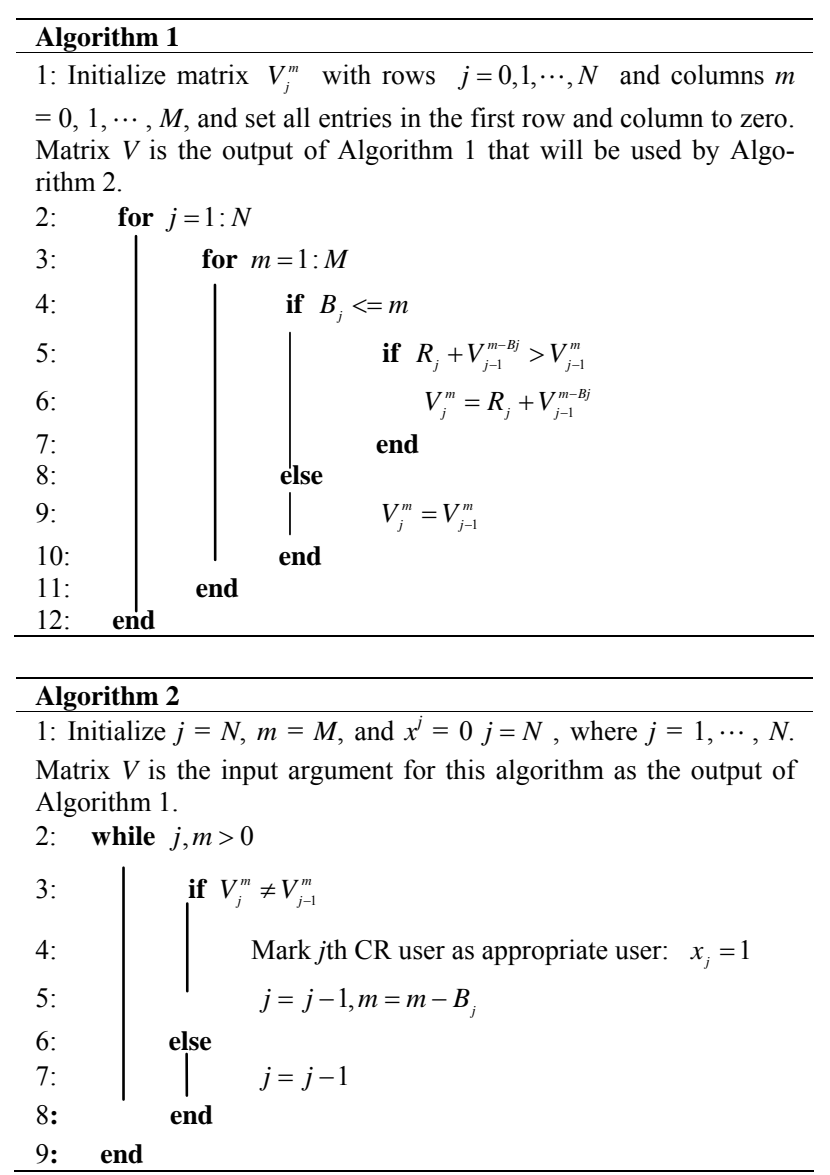




\section{Simulation Results}

To evaluate the performance improvement of the proposed algorithm, extensive simulation results are discussed in this section. We consider a CRN with $N$ CR users in $100 \times 100 \mathrm{~m}^{2}$ field. The CR users are in the three classes $(L=3)$. These are respectively streaming traffic (e.g. data stream download), audio traffic (e.g. mp3) and video traffic (e.g. MPEG1). The percentages of $\mathrm{CR}$ users in these classes are $60 \%, 35 \%$, and $5 \%$ of $N$ CR users, respectively. The required data rates for these three classes are $300 \mathrm{kbps}, 190 \mathrm{kbps}$, and $1.15 \mathrm{Mbps}$, respectively. Also, minimum $B E R s$ are $B E R_{\text {tar }}^{1}=10^{-5}$, $B E R_{\text {tar }}^{2}=10^{-3}$, and $B E R_{\text {tar }}^{3}=10^{-4}$, respectively. The maximum transmitting power of the CR users is $50 \mathrm{~mW}$ and this power mask is enforced to avoid corrupting the connections of PR users. So, for a fair comparison, we let all $\mathrm{CR}$ users use the maximum transmitting power. The signal propagation model in Equations (1) and (2) is used with $n=3$, the maximum linear dimension of transmitter antenna $\left(D_{a}\right)$ is $5 \mathrm{~cm}$, and $d_{0} \approx 1.01 d_{f}$. We set thermal noise, $\sigma_{n}^{2}=-90[\mathrm{dBm}]$ for all spectrum units in the spectrum pool.

We use Jain's fairness index [15] to quantify the throughput fairness of the algorithms. This is a common fairness metric, which is used in network engineering to determine whether users are receiving a fair share of spectrum. Fairness index values closer to 1 indicates better fairness.

We compare our proposed algorithm with DDMAC. DDMAC regardless of optimal solution tries to maximize the overall CRN throughput using distance-dependent algorithm. Also, we compare the proposed algorithm with bandwidth (BW) based and data rate (DR) based algorithms. Their CR user selection strategies are based on low bandwidth requirement and high data rate requirement, respectively. In order to maximize overall CRN throughput, BW based algorithm selects CR users with low bandwidth requirements, such that the number of CR users which share spectrum increases. On the other hand, DR based algorithm selects CR users with high data rates to maximize overall CRN throughput. We compare the performances of these algorithms using simulations.

\subsection{Algorithm Performance with Different Spectrum Pool Sizes}

In Figure 1(a), we compare the overall CRN throughput of the proposed algorithm with other algorithms in different sizes of the spectrum pool, when the number of $\mathrm{CR}$ users is $40(N=40)$. Figure 1(b) shows fairness comparison. These results show that the proposed algorithm achieves a better overall CRN throughput, as well as, a better fairness. It can be observed from these simulation results that as the spectrum pool size increases, the number of CR users who can simultaneously access to spectrum increases, consequently overall CRN throughput and fairness criterion increase. When the spectrum pool size increases to a value more than CRN overall bandwidth requirement, i.e. $\sum_{j=1}^{N} B_{j} \leq M$ (in Figure 1 when $M \geq 200$ ), we don't have any optimization problem and all algorithms have the same performance results. Although in actual wireless network conditions, spectrum pool has insufficient spectrum holes to serve all CR users, so optimal spectrum sharing becomes more important to achieve maximum possible overall CRN throughput. For example, when the spectrum pool has 105 spectrum units (condition that $\sum_{j=1}^{N} B_{j} \geq M$ ), the proposed algorithm achieves $14.88 \%, 6.10 \%$, and $21.11 \%$ higher overall CRN throughput, respectively, compared with those of DDMAC, BW based, and DR based algorithms.

\subsection{Algorithm Performance with Different Numbers of CR Users}

The number of CR users affects the performances of spectrum sharing algorithms. In this section, we consider different numbers of the CR users and a constant spectrum pool size having 100 spectrum units. Figure 2 illustrates the overall throughput and fairness of the CRN in this scenario. As Figure 2(a) shows by increasing the number of CR users, the overall CRN throughput similarly increases in all algorithms. This increase is similar in all algorithms until number of $\mathrm{CR}$ users arises to a value that $\mathrm{CR}$ users' bandwidth requirements is more than common spectrum pool size (number of CR users that saturates spectrum pool). Since, after these number of CR users, spectrum pool is saturated by previous CR users, and DDMAC and DR based algorithms respectively have no idea and good idea to select the best CR users from those added CR users, thus overall CRN throughput stops increasing and becomes nearly fixed, respectively for these two algorithms. In other words, when CR user selection strategy is based on these two algorithms, after saturation point, increasing CR users has insignificant effect on the overall CRN throughput. Whereas, after saturation point, since the proposed and BW based algorithms, respectively have optimal idea and good idea to select CR users from those added CR users, thus the overall CRN throughput continually increases by increasing $\mathrm{CR}$ users. Inasmuch as the proposed algorithm has optimal CR user selection strategy, it has the best operation among other three algorithms for each number of CR users, as Figure 2(a) is shown. Since we assumed available spectrum in spectrum pool was constant, as Figure 2(b) is illustrated, by increasing number of $C R$ users the ratio of $\mathrm{CR}$ users accessing to spectrum decreases, so fairness criterion will be worse by increasing the number of CR users. 


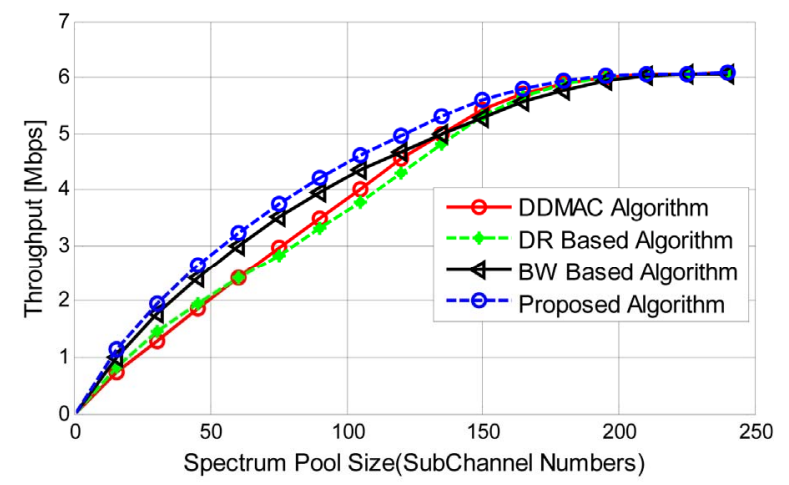

(a)

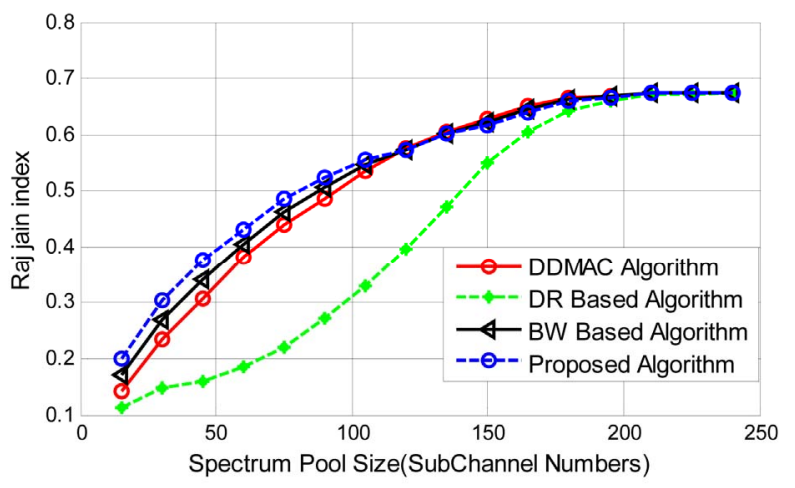

(b)

Figure 1. Algorithm performance with different spectrum pool size. (a) Overall CRN throughput; (b) Fairness criterion.

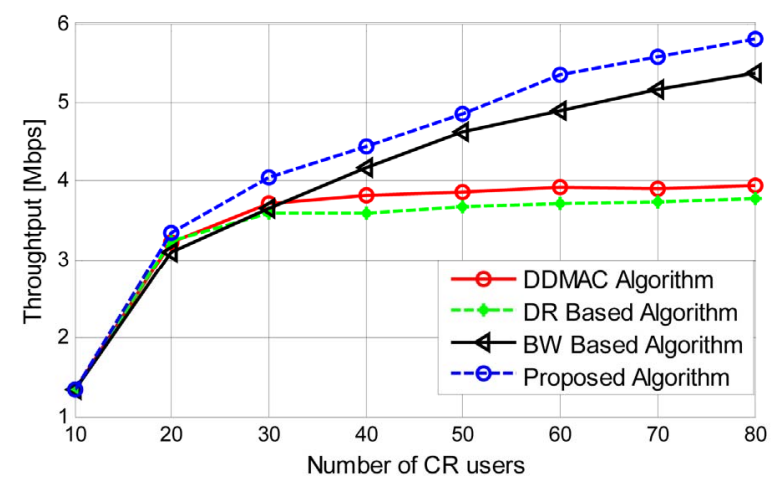

(a)

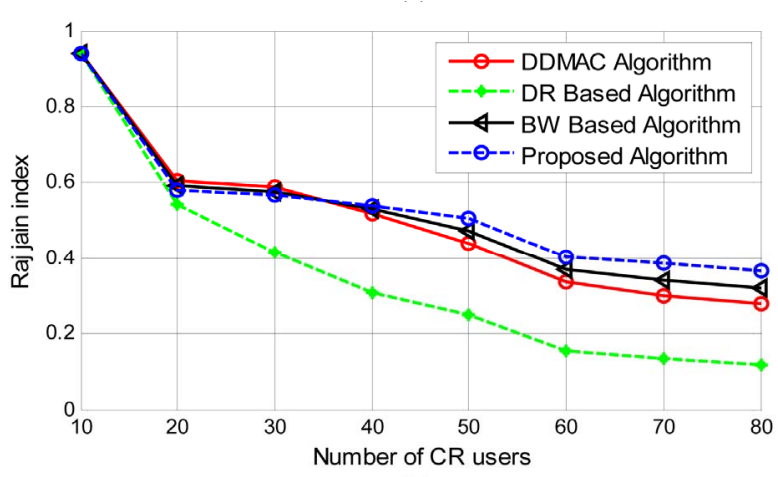

(b)

Figure 2. Algorithm performance with different numbers of CR users. (a) Overall CRN throughput; (b) Fairness criterion.

\subsection{Algorithm Performance with Different Arrival and Departure Event Rates}

Simulation results in Figures 3 to $\mathbf{6}$ show that the performance affected by different arrival and departure event rates. In Figures 3 and 4, we present the overall $\mathrm{CRN}$ throughput and the fairness criterion for different CR user's arrival and departure rates, respectively. We can draw a conclusion that the proposed algorithm significantly improves the overall CRN throughput under these conditions. Also, the proposed algorithm has better performance in fairness view.

Furthermore, the performance comparisons are shown in Figures 5 and $\mathbf{6}$ with different spectrum unit arrival and departure rates, respectively. A larger arrival rate for the spectrum unit or lower departure rate induces availability of more spectrum units; thus, according to the decision strategy of the proposed algorithm, the overall CRN throughput is larger for our proposed algorithm.

\subsection{Algorithm Performance during Time}

In this section, we study the performance of real network conditions by simulating the CRN working for $500 \mathrm{~min}-$ utes under arrival and departure rates given below:

$$
\begin{gathered}
\lambda_{C R U s}=25[\mathrm{CR} \text { user } / 10 \text { minutes }], \\
\mu_{C R U s}=5[\mathrm{CR} \text { user } / 10 \text { minutes }], \\
\lambda_{\text {spec }}=20[\text { spectrum unit } / 10 \text { minutes }], \text { and } \\
\mu_{\text {spec }}=20[\text { spectrum unit } / 10 \text { minutes }] .
\end{gathered}
$$

Since, the proposed algorithm always can optimally select the CR users that have the strongest appropriate to access new available spectrum holes, thus it performs much better than the other algorithms as Figure 7 shows.

\subsection{Algorithm Performance with Different Ratio of Service Type Classes}

Finally, we analyze our proposed algorithm with the different ratios of service types. Let $\alpha^{1}=100 \alpha \%, \alpha^{2}=$ $100(1-\beta) \%$, and $\alpha^{3}=100(\beta-\alpha) \%$ where $\alpha<\beta$ and $0<\alpha, \beta<1$. Simulation results as are shown in Figure 8, illustrate better performances for the proposed algorithm in different classes' ratios. Also, when the traffic ratios in CRN become to pure video traffic, the overall throughput of the CRN increases. In this condition, the CR users that access to spectrum is lower, so fairness criterion becomes lower. 


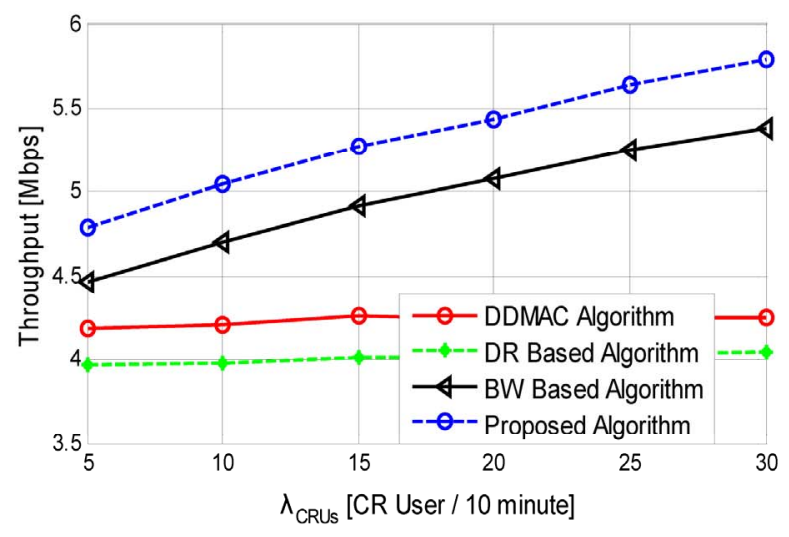

(a)

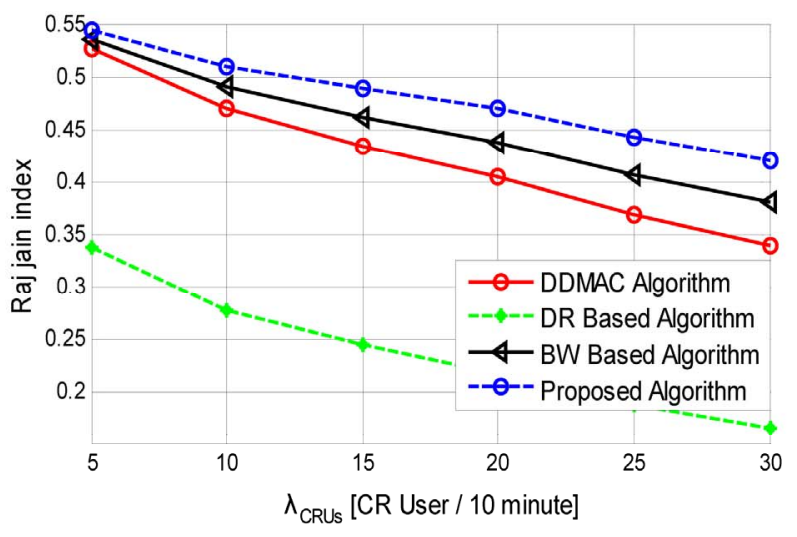

(b)

Figure 3. Algorithm performance with different CR user arrival rates with $\mu_{C R U s}=5$ [CR user/10 minutes], $\lambda_{\text {spec }}=20$ [spectrum unit/10 minutes] and $\mu_{\text {spec }}=20$ [spectrum unit/10 minutes]. (a) Overall CRN throughput; (b) Fairness criterion.

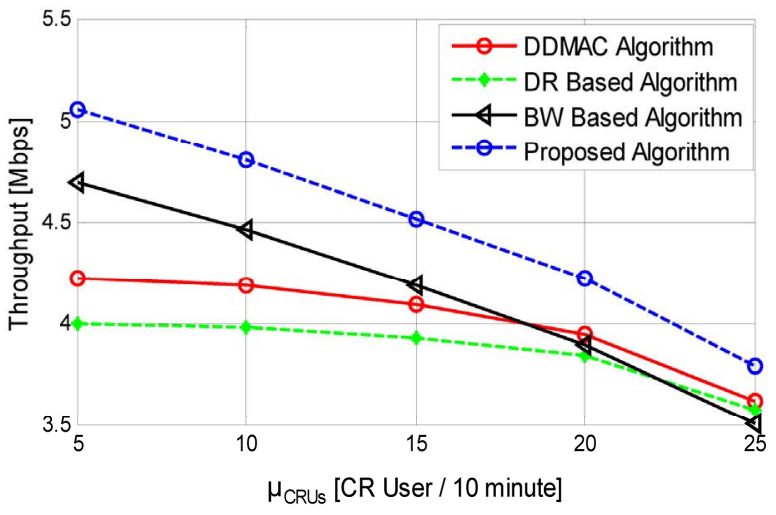

(a)

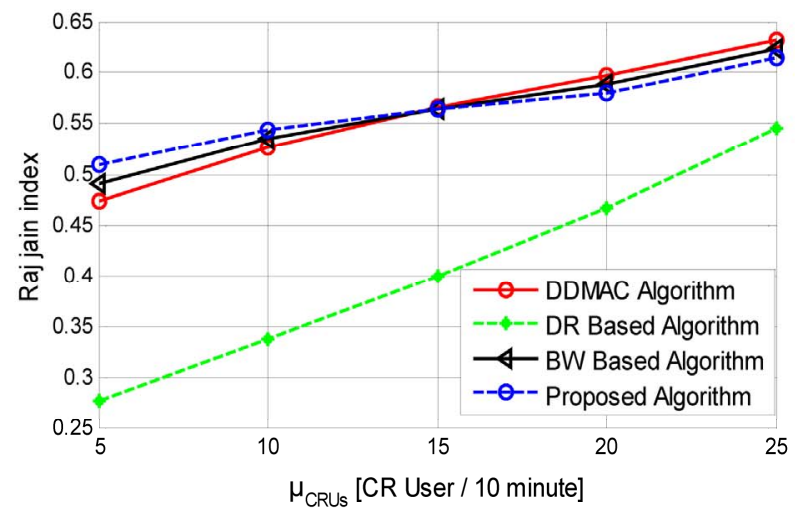

(b)

Figure 4. Algorithm performance with different CR user departure rates when $\lambda_{C R U s}=10$ [CR user/10 minutes], $\lambda_{\text {spec }}=20$ [spectrum unit/10 minutes] and $\mu_{\text {spec }}=20$ [spectrum unit/10 minutes]. (a) Overall CRN throughput; (b) Fairness criterion.

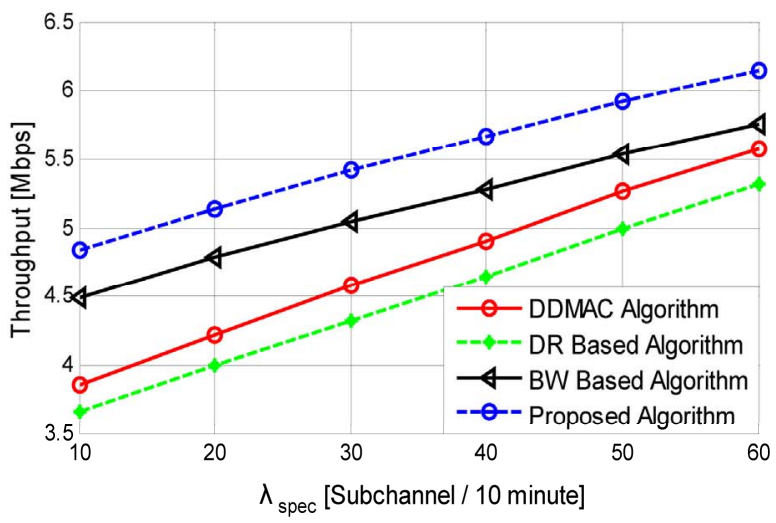

(a)

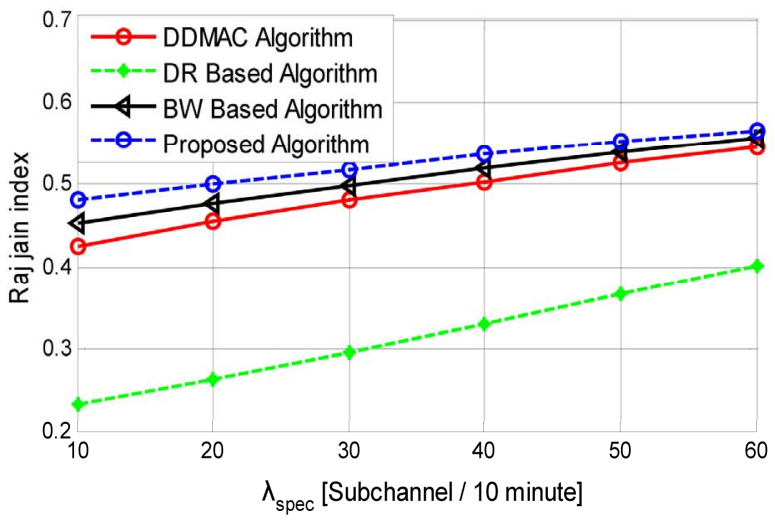

(b)

Figure 5. Algorithm performance with different spectrum unit arrival rates with $\lambda_{C R U s}=15$ [CR user/10 minutes], $\mu_{C R U s}=8$ [CR user/10 minutes] and $\mu_{\text {spec }}=20$ [spectrum unit/10 minutes]. (a) Overall CRN throughput; (b) Fairness criterion.

\section{Conclusion}

In this paper, using log-distance path loss model and $\mathrm{AMC}$, we estimate $\mathrm{CR}$ user bandwidth requirement in different service type classes. Based on the principle of optimality, we proposed a novel cooperative spectrum sharing algorithm with overall CRN throughput objective. This algorithm achieves optimal solution (exhaustive search solution). In addition, its computational complexity $(O(N \times M))$ is much lower than exhaustive search 


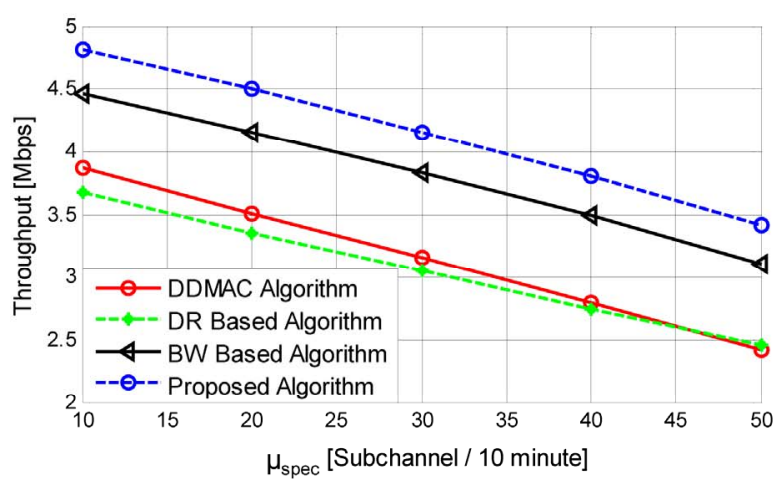

(a)

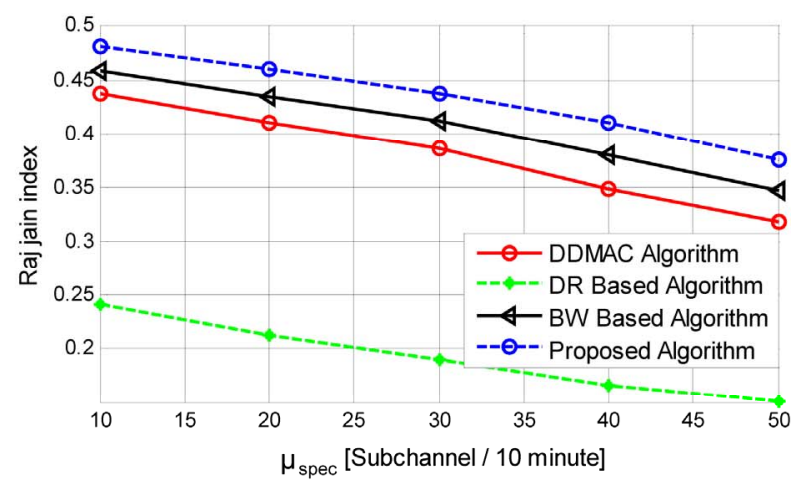

(b)

Figure 6. Algorithm performance with different spectrum unit departure rates when $\lambda_{C R U s}=15$ [CR user/10 minutes], $\mu_{C R U s}=$ 8 [CR user/10 minutes] and $\lambda_{\text {spec }}=10$ [spectrum unit/10 minutes]. (a) Overall CRN throughput; (b) Fairness criterion.

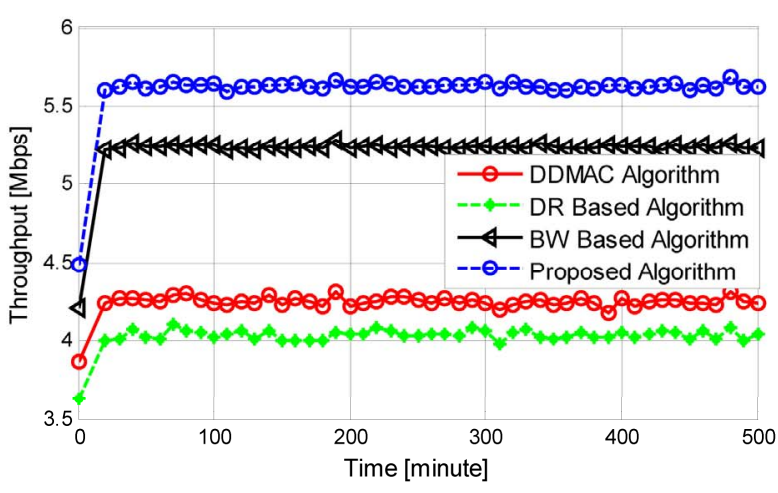

(a)

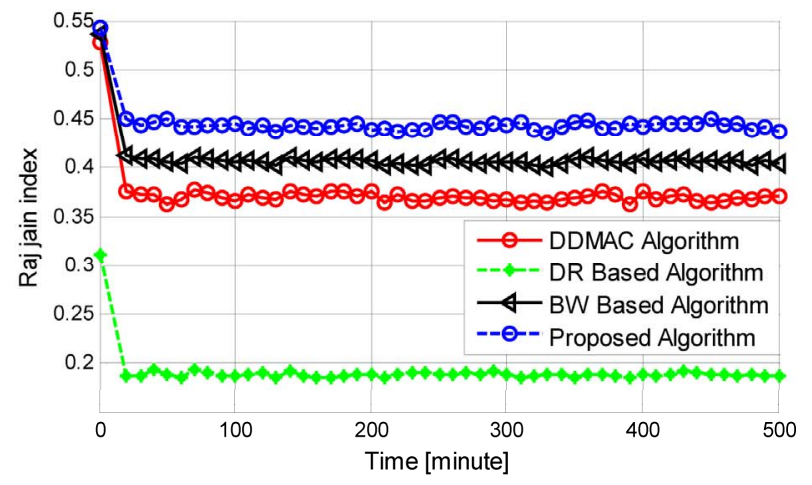

(b)

Figure 7. Algorithm performance during time. (a) Overall CRN throughput; (b) Fairness criterion.

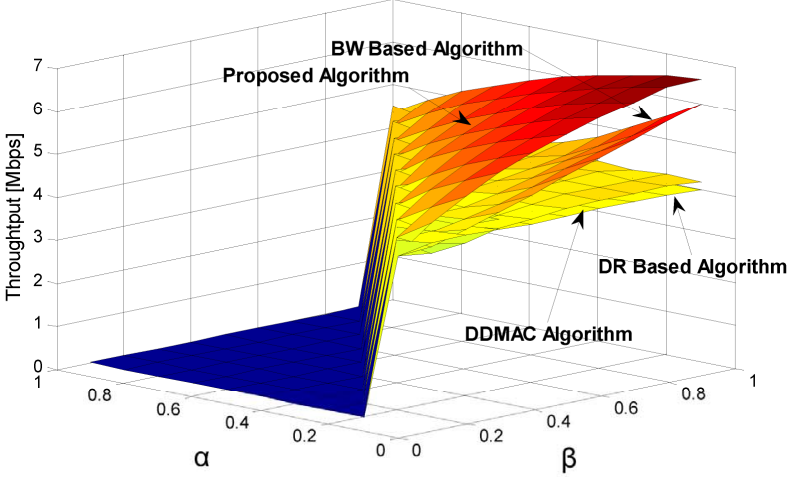

(a)



(b)

Figure 8. Algorithm performance with different percentages of classes where $\alpha^{1}=100 \alpha \%, \alpha^{2}=100(1-\beta) \%$, and $\alpha^{3}=100(\beta$ $-\alpha) \%$, where $\alpha<\beta$. (a) Overall CRN throughput; (b) Fairness criterion.

time complexity. As well, this provides a better result in fairness criterion.

\section{Acknowledgements}

This research is partially funded by the Iranian Institute of information and Communication Technology (the former ITRC). Also, the authors would like to acknow- ledge Mr. Eman Mahmoodi and Mr. Saeed Balaneshin Kordan for their insightful comments and helpful discussions.

\section{REFERENCES}

[1] F. Akyildiz, W.-Y. Lee, M. C. Vuran and S. Mohanty, "Next Generation/Dynamic Spectrum Access/Cognitive 
Radio Wireless Networks: A Survey," Compututer Network, Vol. 50, No. 13, 2006, pp. 2127-2159. doi:10.1016/i.comnet.2006.05.001

[2] Q. Zhao and B. M. Sadler, "A Survey of Dynamic Spectrum Access," IEEE Signal Processing Magazine, Vol. 24, No. 3, 2007, pp. 79-89. doi:10.1109/MSP.2007.361604

[3] I. F. Akyildiz, W.-Y. Lee, M. C. Vuran and S. Mohanty, "A Survey on Spectrum Management in Cognitive Radio Networks," IEEE Communications Magazine, Vol. 46, No. 4, 2009, pp. 40-48. doi:10.1109/MCOM.2008.4481339

[4] T. A. Weiss and F. K. Jondral, "Spectrum Pooling: An Innovative Strategy for the Enhancement of Spectrum Efficiency," IEEE Communications Magazine, Vol. 42, No. 3, 2004, pp. S8-S14. doi:10.1109/MCOM.2004.1273768

[5] P. Si, H. Ji, F. R. Yu and V. C. M. Leung, "Optimal Cooperative Internetwork Spectrum Sharing for Cognitive Radio Systems with Spectrum Pooling," IEEE Transaction on Vehicular Technology, Vol. 59, No. 4, 2010, pp. 1760- 1768.

[6] M. Kaplan and F. Buzluca, "A Dynamic Spectrum Decision Scheme for Heterogeneous Cognitive Radio Networks," Proceedings of 24th International Symposium on Computer and Information Sciences, Guzelyurt, 14-16 September 2009, pp. 697-702.

[7] T. Elkourdi and O. Simeone, "Impact of Secondary MAC Cooperation on Spectrum Sharing in Cognitive Radio Networks," Proceedings of 43rd Annual Conference on Information Sciences and Systems, Baltimore, 18-20 March 2009, pp. 574-578.

[8] B. Bai, W. Chen, and Z. Cao, "Low-Complexity Hierarchical Spectrum Sharing Scheme in Cognitive Radio Networks," IEEE Communications Letters, Vol. 13, No. 10, 2009, pp. 770-772.

doi:10.1109/LCOMM.2009.091383
[9] H. A. B. Salameh, M. Krunz and O. Younis, "Cooperative Adaptive Spectrum Sharing in Cognitive Radio Networks," IEEE/ACM Transaction on Networking, Vol. 18, No. 4, 2010, pp. 1181-1194.

[10] R. Bellman, H. Kagiwada and R. Kalaba, "Dynamic Programming and an Inverse Prolem in Transport Theory," Computing, Vol. 2, No. 1, 1967, pp. 5-16.

[11] T. S. Rappaport, "Wireless Communications-Principles and Practice," 2nd Edition, Prentice-Hall, Saddle River, 2001, pp. 69-100.

[12] H. Sizun, "Radio Wave Propagation for Telecommunication Applications," Springer-Verlag, Berlin, 2005.

[13] J. Zhao, H. Zheng and G.-H. Yang, "Distributed Coordination in Dynamic Spectrum Allocation Networks," Proceedings of IEEE International Syposium on New Frontiers in Dynamic Spectrum Access Networks, Baltimore, 8-11 November 2005, pp. 259-268.

[14] A. Goldsmith and S.-G. Chua, "Variable-Rate VariablePower MQAM for Fading Channels," IEEE Transaction on Communication, Vol. 45, No. 10, 1997, pp. 12181230. doi:10.1109/26.634685

[15] R. Jain, "The Art of Computer System Performance Analysis," Wiley, New York, 1991.

[16] Y. Xing, C. Mathur, M. Haleem, R. Chandramouli and K. Subbalakshmi, "Dynamic Spectrum Access with QoS and Interference Temperature Constraints," IEEE Transaction on Mobile Computing, Vol. 6, No. 4, 2007, pp. 423-433. doi:10.1109/TMC.2007.50

[17] N. Nie and C. Comaniciu, "Adaptive Channel Allocation Spectrum Etiquette for Cognitive Radio Networks," Proceedings of IEEE International Syposium on New Frontiers in Dynamic Spectrum Access Networks, Baltimore, 8-11 November 2005, pp. 269-278. 